Advisory Panel

Jonathan W. Amy

Jack W. Frazer

G. Phillip Hicks
Donald R. Johnson

Charles E. Klopfenstein

Marvin Margoshes
INSTRUMENTATION

Harry L. Pardue

Ralph E. Thiers

William F. Ulrich

\title{
Data Domains-An Analysis of Digital and Analog Instrumentation Systems and Components
}

\begin{abstract}
Data domains concepts offer a means of effectively utilizing new electronic devices which requires only an understanding of basic measurement processes. These concepts can be used to great advantage in designing or modifying systems and in assessing and minimizing the sources of measurement errors.
\end{abstract}

\section{G. ENKE}

Department of Chemistry, Michigan State University,

E. Lansing, Mich. 48823

Giextific instrumentation is being revolutionized by the availability of an ever-increasing array of electronic devices which increase measurement speed, accuracy, and convenience while decreasing instrument size and power requirements. Integrated circuits and hybrid circuits have made many measurement techniques, which were previously only theoretically possible, a reality. The continual decrease in the cost of digital and linear circuits has made many sophisticated devices such as frequency meters, digital $\mathrm{pH}$ meters, signal averagers, and minicomputers practical for most laboratories. As electronic technology continues to advance, we can expect more and more of the sampling, control, and data analysis of scientific measurements to be performed by the instrument itself.

Digital instrumentation has been the scene of much development and interest because of its inherent accuracy capability, convenient numerical output, and potential digital computer compatibility. However, just "digitizing" an instrument does not insure these adrantiges. There are literally hundreds of data handling and digitizing devices available today and an unwise combination of units can actually degrade the output accuracy. Also, many digitized measurement systems, while providing excellent accuracy and convenience, are unnecessarily complicated.

\section{Data Domains Concepts}

The convenience and power of the amazing new electronic devices are irresistible to almost all scientists, but few are in a position to understand these new tools in detail. A means of applying new devices efficiently and effectively, which requires only an understanding of the basic measurement concepts, is needed for most. The data domains concepts described here are very useful in analyzing, describing, modifying, and designing analog, digital, and analog/digital measurement systems and devices and in assessing and minimizing the sources of measurement crrors (1). In addition, a much better understanding of the instrumental data handling process is gained as a result of the study and application of the data domains concept. The first four concepts of data domains analysis are given below:

(1) Measurement data are represented in an instrument at any instant by a physical quantity, a chemical quantity, or some property of an electrical signal. The characteristics or properties used to represent the measurement data can be categorized in groups called "data domains."
(2) As the data proceed through the instrument, a change in the characteristic or property used to represent the measured data is called a "data domain conversion."

(3) All electronic measurement systems can be described as a sequence of two or more data domain converters, each of which can be analyzed separately.

(4) Methods of using electrical signals to represent measurement data fall into three major categories or domains: analog, time, and digital.

Since there are only three data domains for electrical signals, the electronic sections of complex measurement systems can be easily analyzed (or designed) as combinations of only a few basic interdomain converters. Also the hundreds of data handling devices available can be shown to be simply various methods of accomplishing the basic interdomain conversions.

\section{Electronics-Aided Measurement}

In an electronics-aided measurement, the quantity to be measured is converted into an electrical signal and then amplified or otherwise modified to operate a device which visually displays the value of the measured quantity. This process is illustrated for a typical case in the block diagram of Figure 1. An input transducer such as a photodetector, thermistor, glass pH electrode,

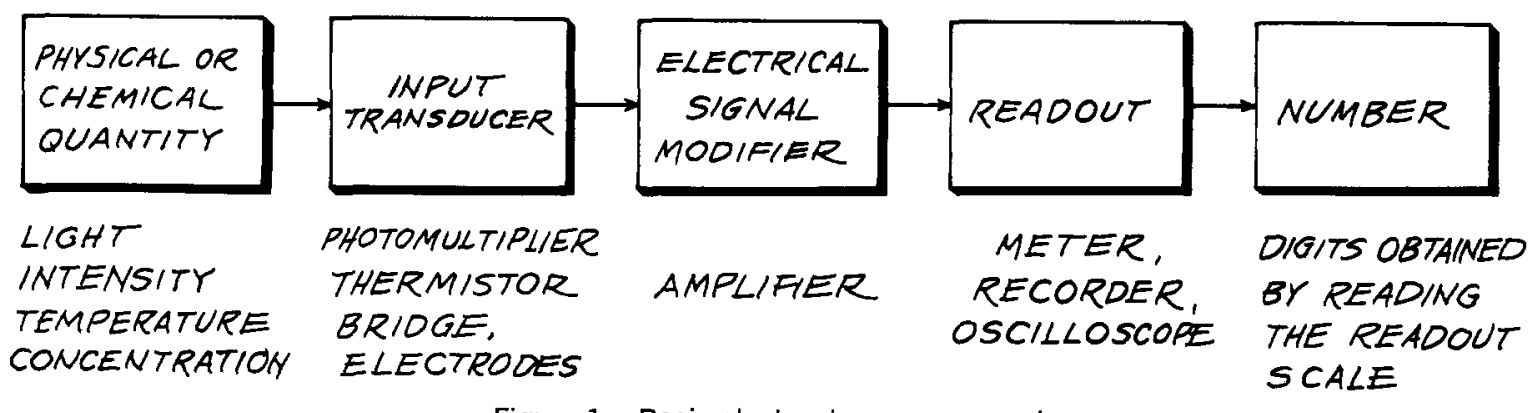

Figure 1. Basic electronic measurement 


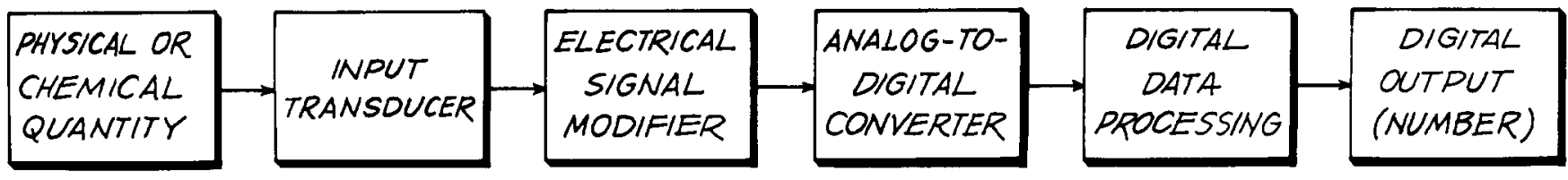

Figure 2. Basic digital electronic measurement

or strain gage is used to convert the quantity to be measured into an electrical signal. The eurrent or voltage amplitude of this signal is related in some known way to the quantity to be measured. The electrical signal from the input transducer is then modified by an electronic circuit to make it suitable to operate a readout device. The electronic circuit is most frequently an amplifier with the appropriate adjustable parameters (zero, standardization, position, etc.) and sometimes with automatic compensation for nonlinearities, temperature variation, etc. of the transducer. The output is a readout device from which a number can be obtained, generally by observing the position of a marker against a numbered scale.

Using the data domains concepts, the basic electronic measurement of Figure 1 is described as follows: The measurement data cxist first as the physical or chemical quantity to be measured. At the output of the input transducer. the measurement data are represented by an electrical signal and are thus in one of the three "electrical" domains. The input transducer is thus a device which converts quantities or translates information from a physical or chemical domain into an electrical domain. The measurement data remain in an electrical domain through the electrical signal modifier. However, the output device converts the electrical signal into some readable form such as the relative positions of a marker and a scalei.e., a nonelectrical domain. Thus the entire measurement can be described in terms of conversions between domains and modifications within domains.

In the basic electronic measurement, at least two converters are required; one to transfer into an electrical domain and one to transfer out. The characteristics of each interdomain converter and each signal modifier affect the quality of the measurement. To take advantage of special input transducers, particular readouts, and available signal processing techniques, an instrument may involve many data domain conversions and signal modifiers. The data domains concept allows each step to be blocked out and analyzed separately. This will be shown to be particularly desirable in assessing sources of errors and the relative ad- vantages of various digitizing or interfacing possibilities.

Digital Measurement. A common form of digital measurement system is shown in block form in Figure 2. At some point after the measurement data have been converted into electrical amplitudes, an analog-to-digital converter is used (2). This is an electronic circuit which converts an analog electronic signal (where the measurement data are represented by the signal amplitude) to a digital electronic signal (which represents integer numbers unambiguously by coded binary-level signals). If the digitization was performed to take adrantage of the great aceurney, power, and versatility of digital data processing, that will be done next. Finally, the numerical binarylevel signal is decoded into a number which is displaved, printed, and/or punched.

Becalse so many advantages are claimed for digital techniques, many techniques have claimed to be "digital." In fact, any type of device which has dial settings or outputs which are numerals in a row is likely to be called digital. $\mathrm{By}$ that standard a decade resistance box is a digital instrument. Since the end result of any measurement is a number, all instruments could be called digital, but the meaning of the word in that sense becomes trivial. Some confine the use of the words "digital instrument" to those instruments which contain binary-level

(a)
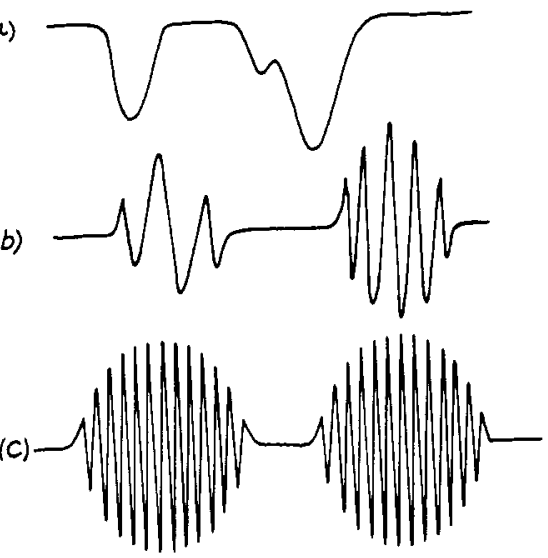

- TIME, WAVELENGTH, FIELO STRENGTH, ETC $\longrightarrow$

Figure 3. Analog $\left(E_{A}\right)$ domain signals electronic logic circuits of the type developed for digital computers. As will be shown later, it is common for measurement data to be represented by a binary-level electronic signal and still not be "digitized" or numerical. Therefore, in this paper, a digital instrument will be defined as one that uses a digital electronic signal to represent the measurement data somewhere within the instrument. The analysis and design of digital measurement systems necessarily involves an understanding of the ways electrical signals can represent data and how conversions from one form to another are accomplished.

\section{Electronic Data Domains}

There are only three basic ways by which measurement data are represented by an electrical signal: Analog, simbolized $E_{A}$, in which the amplitude of the signal current or voltage is related to the data; time, $E_{\Delta t}$, in which the time relationship between signallevel changes is related to the measurement data: and digital, $E_{D}$, in which an integer number is represented by binary-level signals. The characteristic signals in each of these domains and examples of their use are described in this section.

Analog, $E_{\mathrm{A}}$. The measurement data in this domain are represented by the magnitude of a voltage or a current. The analog domain signal is continuously rariable in amplitude. Also, the analog amplitude can be measured continuously with time or at any instant in time. Most input transducers used todily convert the measurement dati from the phrsical and chemical domains $(P)$ to the $E_{\text {A }}$ domain. Examples of $P$-to$E_{A}$ converters are: photodetectors which convert light intensity to an electrical current, a thermistor bridge which converts temperature difference to an electrical potential, a combination $\mathrm{pH}$ electrode which converts solution acidity into an electrieal potential, and a flame ionization detector which converts the concentration of ionizable molecules in a gas into an electrical current. Figure 3 shows some typical $E_{\text {A }}$ signals.

At ench instant in time, the mensured qunntity is represented by a signal 
amplitude. The variations in the signal amplitude may be plotted against time, wavelength, magnetic field strength, temperature, or other experimental parameters as shown in Figure 3. From such plots, additional information can often be obtained from a correlation of amplitudes measured at different times. Such information would include simple observations like peak height, peak position, number of peaks, or more complex correlations such as peak area, peak separation, signal averaging, and Fourier transformation. The techniques of correlating data taken at different times must be distinguished from the techniques of converting the data taken at each instant into a usable form. It is the latter problem that this paper is primarily concerned with. The former problem is handled by data processing techniques, once the required instantaneous data points have been converted to a useful form and stored.

Signals in the $E_{A}$ domain are susceptible to electrical noise sources contained within or induced upon the circuits and connections. The resulting signal amplitude at any instant is the sum of the data signals and the noise signals.

Time, $E_{\Delta t}$. In this domain, the measurement data are contained in the time relationship of signal variations, not the amplitude of the variations. Typical $E_{\Delta t}$ domain signals are shown in Figure 4. The most common $E_{\Delta t}$ domain signals represent the data as the frequency of a periodic waveform (a), the time duration of a pulse (b), or as the time or average rate of pulses (c). These are logic-level signals-i.e., their signal amplitude is either in the HI or 1 logic-level region or the LO or $\theta$ logic-level region. The data are contained in the time relationship between the logic-level transitions. The greater the slope $(d E / d t)$ of the signal through the logic-level threshold region, the more precisely the transition time can be defined. Because the data in an $E_{\Delta t}$ domain signal are less amplitude-dependent than in an $E_{A}$ domain signal, they are less affected by electrical noise sources. A common example of this is the F.I radio signal ( $E_{\Delta t}$ domain) vs. the more noise susceptible AMI radio signal ( $E_{A}$ domain). The greater the difference between the average 0 or 1 signal-level amplitude and the logic-level threshold, the less susceptible the signal will be to noise-induced error. In these respects, the signal shown in Figure $4 \mathrm{~b}$ is better than those of Figures $4 \mathrm{a}$ and $4 \mathrm{c}$. The logic-level transitions of signals like Figures $4 a$ and $4 c$ are generally sharpcned to those like $4 \mathrm{~b}$ before the sig- (a) - $-A-A-A H A-A-A-\frac{11}{10}$

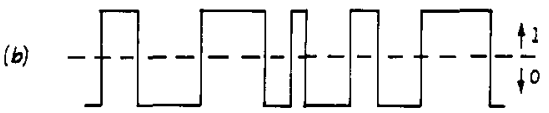

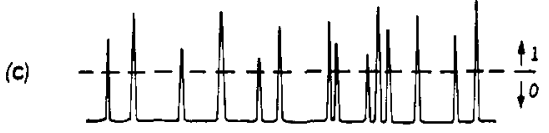

TIME

Figure 4. Time $\left(E_{\Delta t}\right)$ domain signals

nificant time relationship is measured. This is accomplished by a comparator or Schmitt trigger circuit. Examples of converters producing $E_{\Delta t}$ domain ignals from physical domain quantities are: a cristal oscillator that produces a temperature-dependent frequency because of the temperature characteristics of the quartz crystal, an oscillator which has an output frequency dependent upon the value of the capacitance used in the oscillator circuit, and the Geiger tube which converts level of radioactivity to a pulse repetition rate. An example of a $E_{A}$-to- $E_{\Delta t}$ domain converter is a voltage-controlledoscillator or voltage-to-frequencr converter which provides an output frequency related to an input voltage.

The $E_{\Delta t}$ domain signal, like the $E_{A}$ domain signal, is continuously variable since the frequency or pulse width can be varied infinitesimally. However, the $E_{\Delta t}$ signal variable cannot be measured. continuously with time or at any instant in time. The minimum time required for conversion of an $E_{\Delta t}$ domain signal to any other domain is one period or one pulse width.

Digital, $E_{D}$. In the digital domain, the measurement data are contained in a 2-level signal ( $\mathrm{HI} / \mathrm{LO}, 1 / 0$, etc.) which is coded to represent a specific integer (or character) (3). The digital signal may be a coded series of pulses in one channel (serial form) or a coded set of signals on simultaneous multiple channels (parallel form). Representative digital signal waveforms are shown in Figure 5 . The count serial waveform (a) is a series of pulses with a clearly defined beginning and end. The number represented is the number of pulses in the series. The count serial waveform of Figure 5 might represent, for instance, the number of photons of a particular energy detected during a single spark excitation. The count serial form is simple but not very efficient. To provide a resolution of one part per thousand, the time required for at least one thousand pulses to nceur must be allowed for each series of pulses.

The most efficient serial digital signal is the binary-coded serial signal shown in Figure 5b. In this signal, each pulse time in the series represents a different bit position in a binary number. The appearance of a pulse at a time position indicates a 1 ; the absence of a pulse, a 0 . The data are not represented by the exact time of the pulse as in the $E_{\Delta t}$ domain, but by the signal logic level present within a given time range. The binary number represented by the wareform shown is 101101011 which is decimnl 36.3. A series of $n$ pulse times has a resolution of one part in $2^{n}$. Thus a 10-bit series has a resolution of one (a)

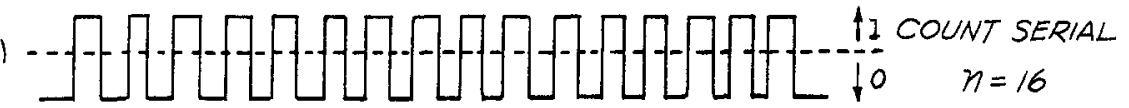

(b)

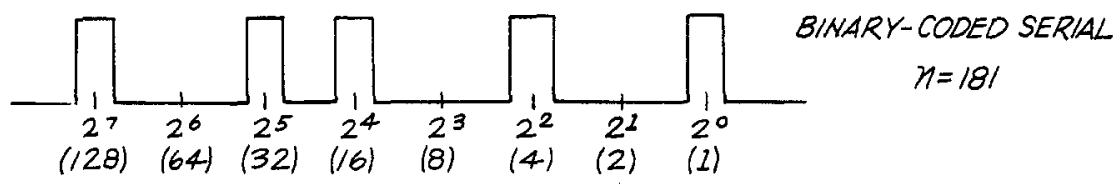

(C)

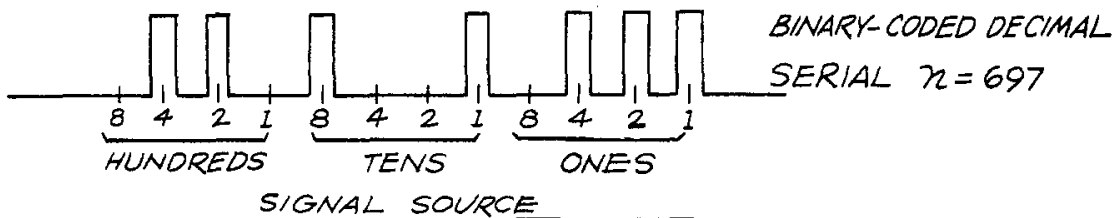

(d)

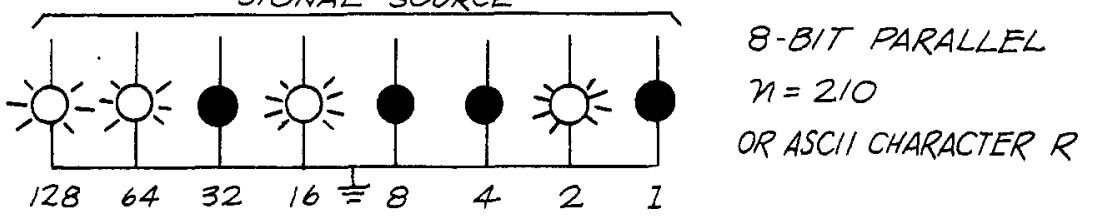

Figure 5. Serial and parallel digital signals 


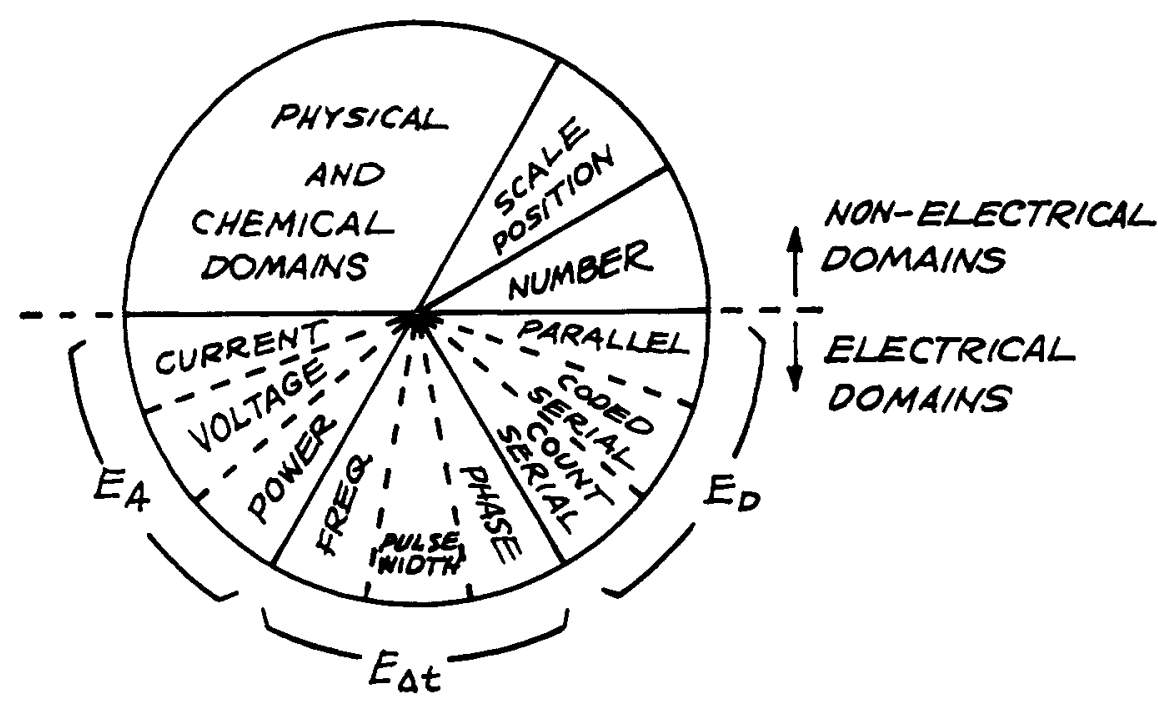

Figure 6. Data domains map

part in $2^{10}=1024_{10}$, and a 20 -bit series has a resolution of better than one part per million.

The binary-coded decimal serial form is somewhat less efficient but very convenient where a decimal numerical output is desired. Each group of four bits represents one decimal digit in a number. Twelve bits can thus represent three decimal digits and provide one part in one thousand resolution

A parallel digital signal uses a separate wire for each bit position instead of a separate time on a single wire The principal advantage of parallel digital data connections is speed. An entire "word" (group of bits) can be conveyed from one circuit to another in the time required for the transmission of one bit in a serial connection. An 8 -bit parallel data source is shown in Figure $5 \mathrm{~d}$ connected to indicator lights to show the simultaneous appearance of the data logic levels on all eight data lines. Binary coding (shown), binarycoded decimal coding, and others are used for parallel digital data. Parallel data connections are used in all modern, fast computers. Serial data connections are often used for telemetry and slow computer peripherals such as teletypes.

\section{Mapping Domain Conversions}

It has been pointed out that electronic instruments making chemical or physical measurements use no fewer than two data domain conversions. In fact, modern laboratory instruments frequently use three or more domain conversions to perform the desired measurement. Knowing the data domains involved in a particular instrument's operation can help in understanding its operation, applications, limitations, and adaptability as part of a larger measure- ment system. When analyzing an instrument it is helpful to use the data domains "map" shown in Figure 6. The path of the signal can be traced out on the map as it is followed through the instrument. This process will be illustrated for several chemical instruments of various types.

$p H$ Meter. The block diagram of a conventional $\mathrm{pH}$ meter is shown in Figure 7. The combination glass/calomel electrode converts the hydrogen ion activity (chemical composition domain) to an electrical potential $\left(E_{A}\right.$ domain). This signal is amplified and converted to a current amplitude that is used to deflect the meter pointer. A

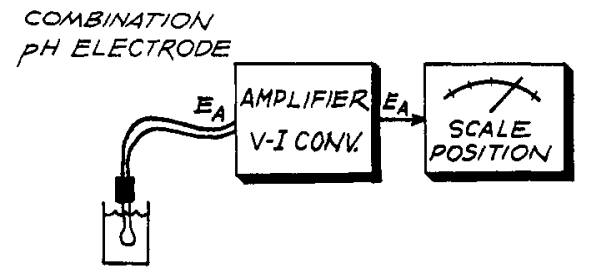

Figure 7. $\mathrm{pH}$ meter
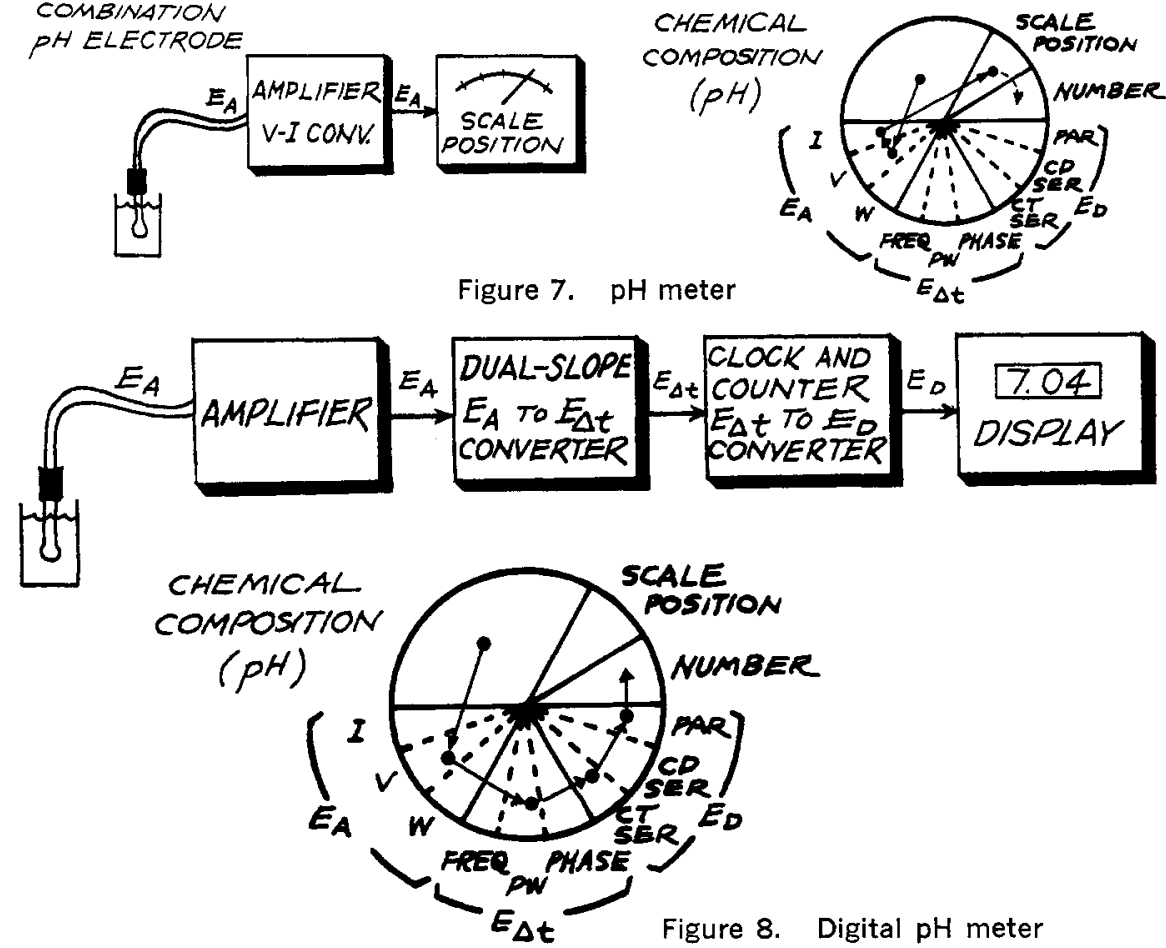

Figure 8. Digital pH meter 


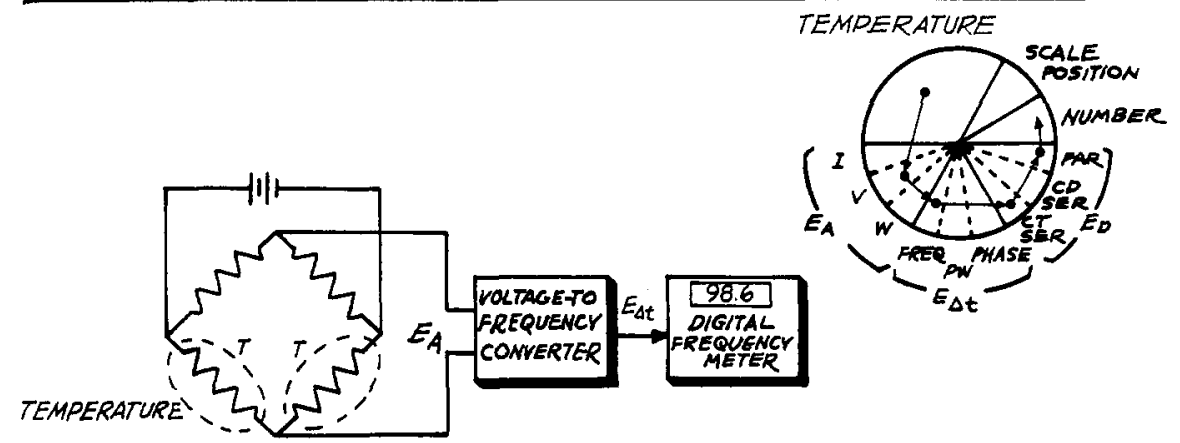

Figure 9. Thermistor-digital temperature measurement
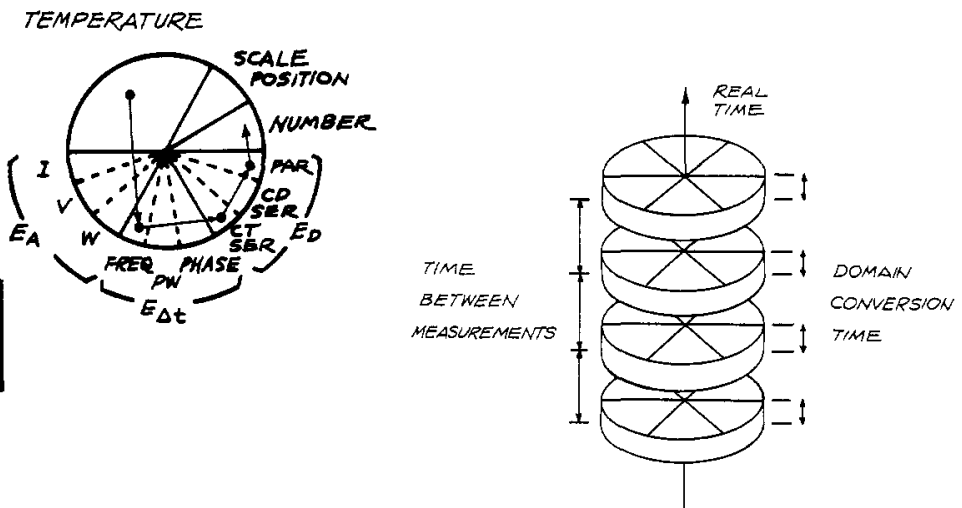

Figure 10. Crystal oscillator temperature measurement

Figure 11. Successive domain conversions

The count serial signal is in turn converted to parallel digital for the display by a counter. From the domains map it is seen that four interdomain and one intradomain conversions are involved in the measurement.

Digital Temperature Measurement. Two approaches to a digital thermometer are compared here. The first is a thermistor bridge connected to a digital voltmeter $\left(E_{D}\right)$ as shown in Figure 9 . The thermistor bridge circuit converts temperature to voltage. In this case the $E_{A}$-to- $E_{D}(\mathrm{~A} / \mathrm{D})$ conversion is accomplished by a voltage-to-frequency converter and a frequency meter. The digital frequency meter operates by counting the number of cycles of an input signal that occur in a specific time. The resulting domain path is shown. There are four interdomain and one intradomain conversions.

The second approach is the use of a quartz crystal which has a temperature-dependent resonant frequency. An oscillator is used to convert the resonant frequency to an electrical signal in the $E \Delta t$ domain. The block diagram is shown in Figure 10. A mixer is used to subtract a standard frequency, $f_{s}$, from the temperature-dependent frequency, $f_{t}$, to obtain a signal for which the frequency and temperature are related directly. This is an example of signal modification occurring in the $E_{\Delta t}$ domain. Note that this digital thermometer requires one less interdomain conversion than that of Figure 9. Whether

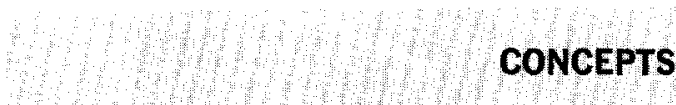

\section{Data Domains}

1. Measurement data are represented in an instrument at any instant by a physical quantity, a chemical quantity, or some property of an electrical signal. The characteristics or properties used to represent the measurement data can be categorized in groups called "data do mains."

2. As the data proceed through the instrument, a change in the char acteristic or property used to represent the measured data is called a "data domain conversion."

3. All electronic measurement systems can be described as a sequence of two or more data domain converters, each of which can be analyzed separately.

4. Methods of using electrical signals to represent measurement data fall into three major categories or domains: analog, time, and digital.

\section{Measurement Devices}

5. All measurement devices employ both a difference detector and a reference standard quantity.

6. Either the difference detector or the reference standard can affect the accuracy of the measurement.

7. The reference standard quantity is the same property or characteris tic as that which is being measured.

8. Interdomain converters have the characteristics of measurement de vices.

this simplification would result in greater accuracy, however, depends upon the accuracy of the converters involved in each case.

\section{Conversions of Varying Quantities}

The examples used in the previous section were measurements of steady- state quantities which were not expected to vary perceptibly over the interval of measurement. When the time variation of quantities in the various data domains is considered, a third dimension (time) needs to be added to the data domain map of Figure 6 , as shown in Figure 11. Here each interdomain conversion is shown as a slice 
Advertising removed

from this page 


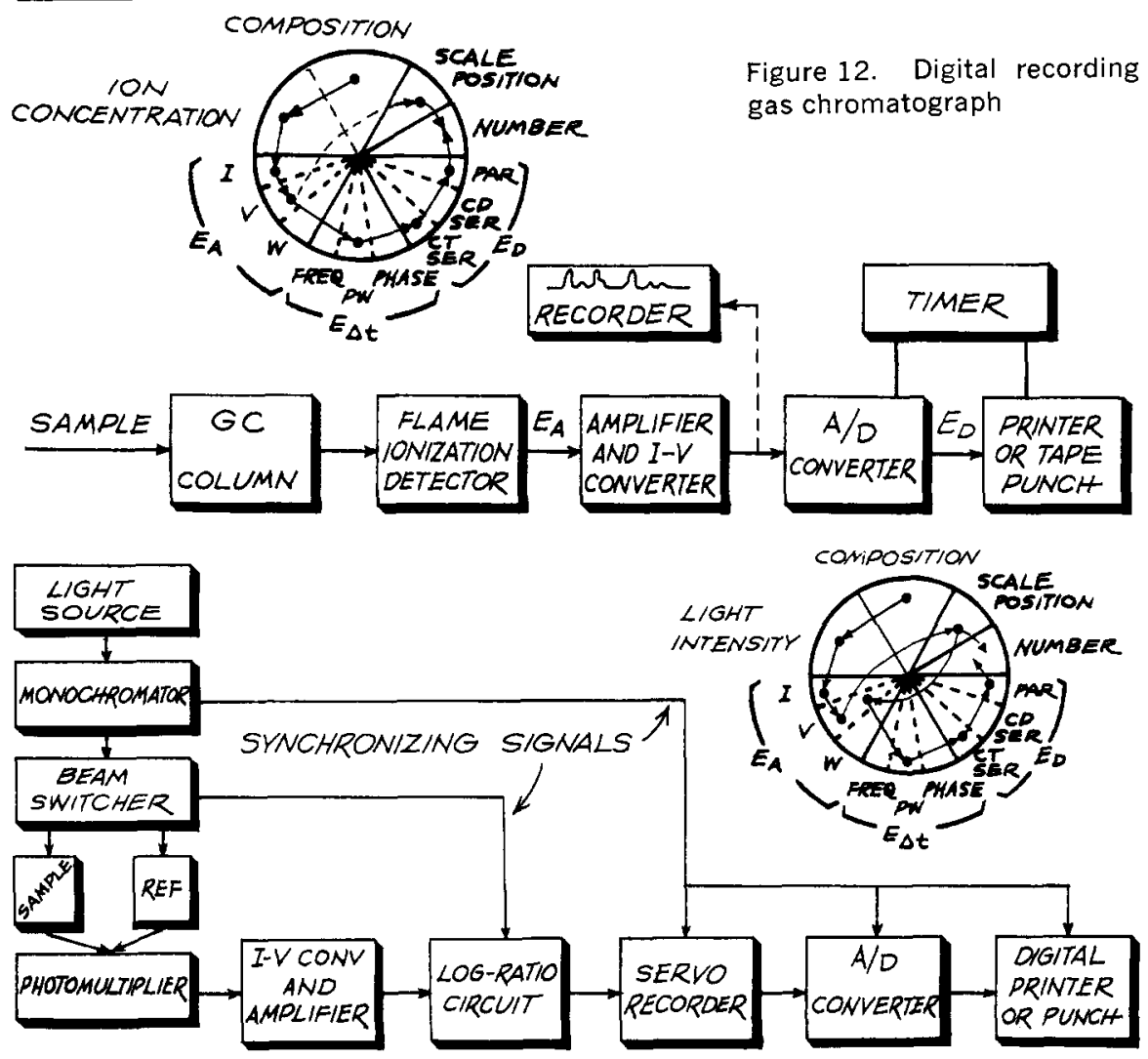

Figure 13. "Digitized" double-beam spectrophotometer

\section{COMPOSITION}

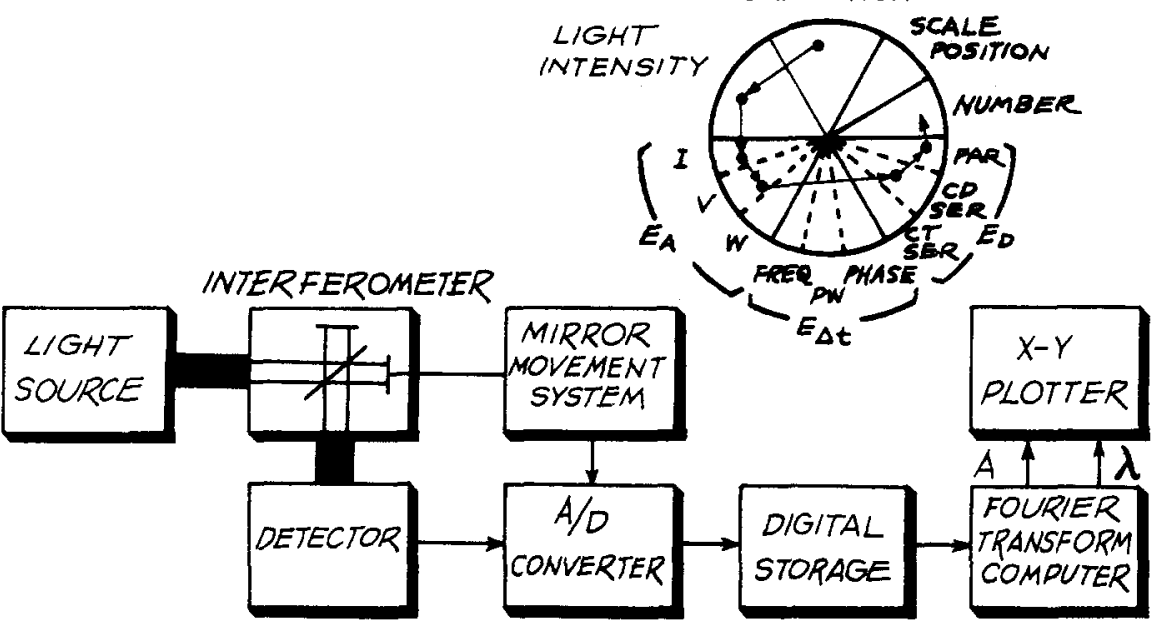

Figure 14. Fourier transform spectrometer

across the real-time continuum. If a quantity that varies continuously with time is to be converted to the digital domain, the resulting number can only be true for a specifie instant in time. It is not possible, therefore, to make a truly continuous digital record of a varying quantity. What can be done is to measure the varying quantity at successive instants in time. The numerical result of ench measurement is then stored in order in memory registers, or recorded on punched cards or paper tape, or by magnetic recording devices. If the measurements are made frequently cnough for the varying quantity to change only slightly between cach time, the digital record can quite accurately represent the amplitude $v s$. time behavior of the measured quantity. Of course, the maximum frequency of measurement is limited by the time required to convert the measured quantity into the digital domain and record it. Successive data domain conversions will be illustrated by three examples of digitized measurement systems.

Gas Chromatography. A digital recording gas chromatograph is shown in Figure 12. The components in the sample mixture are separated by the column resulting in a flow of gas of varying composition through the flame ionization detector. The flame in the detector converts the hydrocarbon concentration into an ion concentration (an intradomain conversiony which is then converted by the electric field in the detector to an electrical current (an interdomain conversion). The current signal is amplified and converted to a voltage amplitude suitable for $A / D$ conversion and/or recording. The desired data are a record of detector current $v s$. time. The time relationship of the printed or punched values of the current amplitudes is generally obtilined by having the successive $A / D$ conversions performed at precise and regular time intervals. This is accomplished by the timer shown in the block dingram. The domain path for a single conversion (one time slice) is also shown.

Absorption spectrophotometry. The block diagram of a digitized recording double-bcam spectrophotometer is shown in Figure 13. A narrow wavelength range of light from the light source is selected by the monochromator and passed on to the beam switcher and cell compartment. The beam switcher alternately directs the monochromatic beam through the reference and sample cells to the photomultiplier tube detector. This produces an electrical current ( $E_{A}$ domain) which has an amplitude alternating between sample and reference beam intensities $P$ and $P_{0}$. The desired output signal for the recorder is absorbance $A=\log _{10}$ $\left(P_{0} / P\right)$, which is accomplished in the log-ratio circuit. This circuit performs a correlation between signal levels measured at two different times. It must, therefore, have a memory and a synchronizing connection to the beam switcher. The recorder is to plot absorbance $v s$, the wavelength of light from the monochromator. The recorder chart drive thus has a synchronizing connection to the monochromator wavelength drive mechanism.

This standard spectrophotometer was later digitized by putting a retransmitting pot assembly on the servorecorder. This converts the recorder pen position to a roltage amplitude which is connceted to an $\mathrm{A} / \mathrm{D}$ converter and printer or punch. Since the absorbance value recorded for precise wavelength (rather than time) intervals is desired, the $A / D$ converter and printer are synchronized to the wavelength drive mechanism rather than a timer.

The data domains map for the resulting instrument is shown in Figure 13. It contains nine conversions; seven interdomain and two intradomain. The excursion in to the scale position domain is unneccseiry to the digitizing process and suggests that the $A / D$ converter would have been better connected to the log-ratio circuit output, if possible. It is interesting to note that if photon 


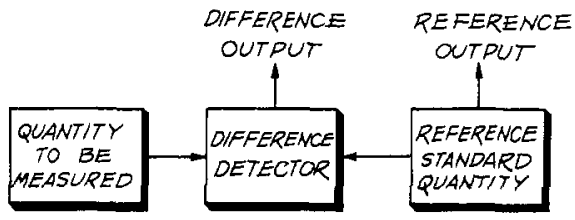

Figure 15. Basic measurement sys. tem

counting is used to measure the relative intensities, the number of interdomain conversions is reduced to three and a digital log-ratio circuit is required.

Fourier Transform Spectroscopy. A Fourier transform spectrometer is an example of a conceptually very simple data acquisition system connected to a complex data correlating and processing system. The block diagram is shown in Figure 14. To obtain the interference pattern, the $\mathrm{A} / \mathrm{D}$ converter converts the detector output signal at constant increments of movement of the reference beam mirror. Each piece of data is stored for use in the Fourier transform calculation. The data domains man as shown is complete for each piece of data as it is acquired and stored. A successive approximations type of $\mathrm{A} / \mathrm{D}$ converter has been assumed in this map. There are four interdomsin and two intradomain conversions in all. After the transform calculation (which involves an intereorrelation of all the measured data points) is complete, a plot of absorbance vs. wavelength can be made.

This example demonstrates that Fourier transformation from amplitude vs. time or space to amplitude vs. frequency (or the reverse) is really an intercorrelation of analog signals which have already been "mensured." It is essontial to clistinguish between the data domains involved in the methods of acquiring each data point and the methods of correlating and displaying groups of data points.

\section{Errors in Domain Conversions}

To understand the sourees of error in domain conversion, it is helpful to review briefly the basic measurement process. Mensurement can be defined as: The determination of a particular characteristic of a sample in terms of a number of standard units for that characteristic. The comparison of the quantity to be measured with standard units of that quantity is implicit in this definition. The comparison concept in measurement is illustrated by Figure 15 . The quantity to be measured is compared with a reference standard quantity. The difference is converted to another form (domain) such as scale position. The quantity measured is then the sum of the standard units in the reference quantity and the difference output calibrated in the same standard units.

All measurement devices involve both a difference detector and a reference standard, although they differ widely in the degree to which one or the other is relied upon in the measurement. As an example, three mass measuring devices can be compared in this regard. With a double pan balance, the unknown mass is compared with standard weights, whole units and fractional, until the difference detector (beam pointer) points to zero. In this case, the accuracy and resolution of the standard weights determines the accuracy of the measurement as long as the difference detector is sufficiently sensitive. To accuracy requirement is placed on the off-null calibrations of the difference detector. The other extreme is a spring-loaded scale, such as a fish or bathroom scale. In this case, the refrence standard weights are used to calibrate the scale markings of the manufacturer's original prototype. In use, the measurement accuracy depends entirely upon the off-null markings on the scale. The reference standard quantity compared by the scale in this case is zero weight. In between these two extremes is the single pan balance with an optical scale for fractional weights. Balances of this type rely upon accurate stundard weights for the most siguificant figures and upon off-null calibrations for the less significant figures.

Similar comparisons and analyses can he made for other tipes of measurement derices. A potentiometric voltage measurement depends much more upon the slidewire (standard voltage unit adiustment) calibration than upon the galvanometer null detector, while an clectrical meter depends much more upon the difference detector calibrations than upon the standard. This kind of analysis is helpful in assessing the sources of errors in measurement devices and in choosing among available devices for a particular application. After considering a variety of men-urement systems in this way, some basic concepts concerning measurement derices erolve which can be added to the four data domains concents listed carlier.

(5) All measurement devices employ both a difference detector and a reference standind quantity.

(6) Either the difference detector or the reference standard can affect the accuracy of the measurement.

(7) The reference standard quantity is the same pronerty or characteristic as that which is being measured. 
Advertising removed

from this page 
A data domain conversion is the conversion of a number of units of some physical, chemical, or electrical characteristic into a related number of units of a different characteristic; for instance, the conversion of units of $\mathrm{pH}$ into Nernst factor potential units by a combination $\mathrm{pH}$ electrode. Devices for converting data from one domain to another are "measuring" one characteristic in terms of another. Therefore,

(8) Interdomain converters have the characteristics of measurement devices.

Using a combination $\mathrm{pH}$ electrode as an example of an interdomain converter to illustrate concepts 5-7: 5) The combination $\mathrm{pH}$ electrode itself is the difference detector; the reference standard is the standard buffer solution used to "standardize" the voltage output at a given $\mathrm{pH}$. 6) The conversion error (difference between the predicted and actual potential $/ \mathrm{pH}$ relationship) depends upon the accuracy of the standard solution and upon the accuracy of the electrode response. The greater the $\mathrm{pH}$ difference between the standard and unknown solutions, the more the conversion accuracy depends upon the electrode's characteristics. 7) The reference standard is $\mathrm{pH}$, the units which are being converted to electrical potential.

Once one is accustomed to looking for the difference detector, reference standard, and accuracy dependence of interdomain converters, the basis of the conversion and the sources of error are easier to uncover. Every A/D converter contains a standard voltage or current source and every $\Delta t / D$ converter contains a standard clock oscillator, as expected from concept 7 above. In both cases the conversion accuracy depends directly upon the standard sources and, for various types, to a greater or lesser degree upon the other converter characteristics.

Conversions of data within domains can often be accomplished with high accuracy by simple transfer devices that require no comparison or reference standard. For instance, a resistor can be used to convert current to voltage or vice versa; the shift register will convert between serial digital and parallel digital domains.

\section{Domain Converter Classification}

A classification scheme for data conversion devices would seem desirable for two purposes: to categorize by function the great many devices available, and to provide a way to organize these devices in to complete measurement systems. It is natural and useful to classify converter devices according to the domains which the device converts from 
Advertising removed

from this page 


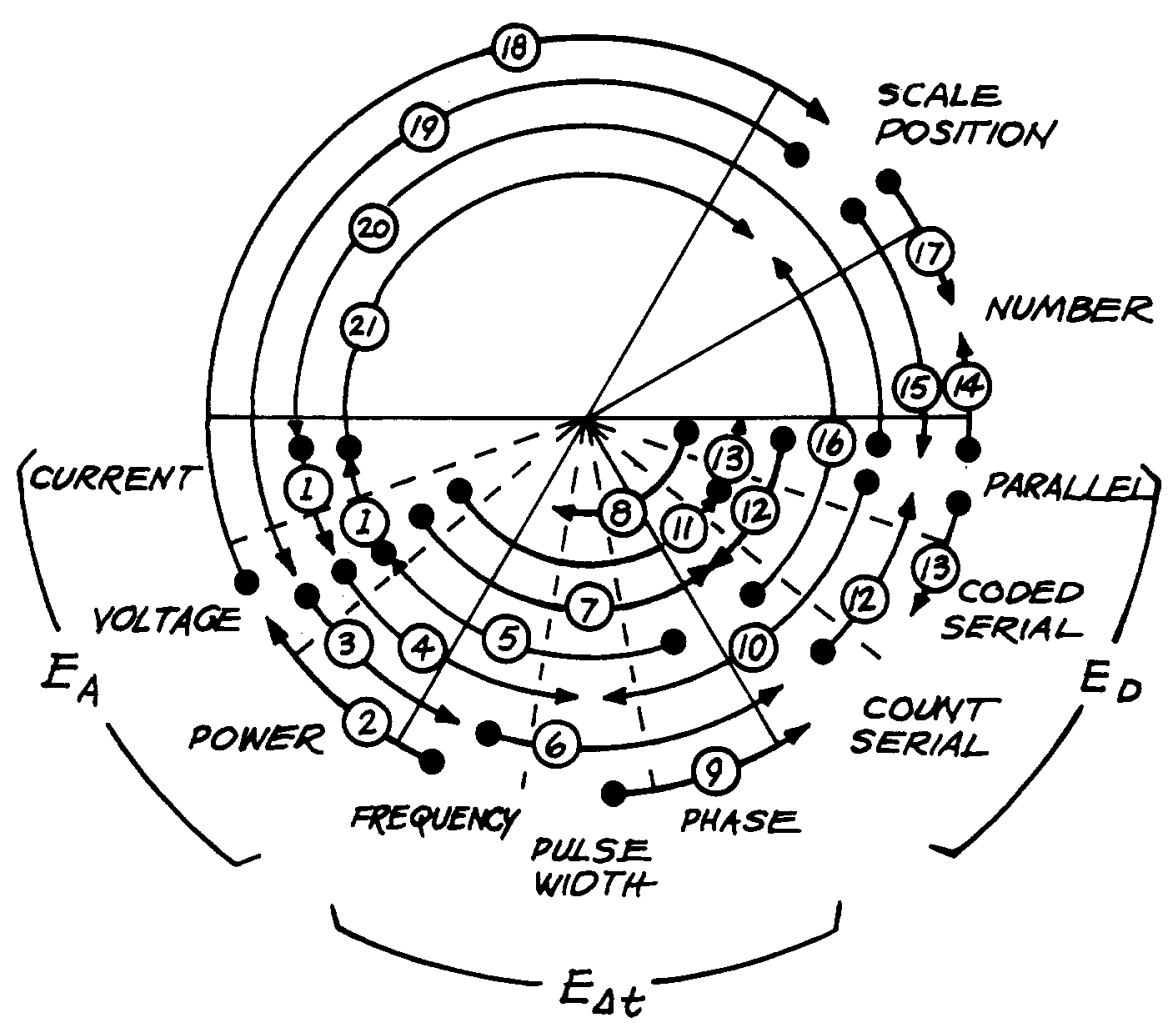

Figure 16. Domain converter classification map
1. Resistor, op amp circuit

2. Count rate meter

3. V.F converter

4. Dual slope $A / D$ converter, ramp $A / D$ converter

5. Phase angle meter

6. Counting gate timer

7. Staircase A/D converter

8. Programmable frequency divider

9. Gated oscillator

10. Preset digital timer

11. Successive approximation $A / D$ converter
13. Shift register

14. Nixie tubes, printer

15. Shaft rotation encoder

16. Stepper motor

17. Mechanical shaft turns counter

18. Recorder

19. Retransmitting potentiometer

20. D/A converter (ladder or weighted sum)

21. Meter
12. Counter

path which has the fewest conversions should also be apparent from tracing the possible routes shown in Figure 16. However, the shortest path is not always the path of choice. For example, to go from scale position to parallel digital, the direct path is 15 . However, absolute shaft rotation encoders with a high accuracy and ruggedness requirement could cost much more than the devices needed to take route $19-7-12$.

\section{Summary}

The data domains and measurement concepts discussed here can be used to great advantage to analyze and describe available analog/digital instruments, to design or modify measurement systems, and to determine the sources of measurement errors. The data domains map can serve to show the data path from a block diagram or to devise a possible block diagram for instruments or modules, knowing only the input and output domains.

To analyze or describe an instrument use the instrument description and block diagram to carefully follow the measurement data step-by-step through the instrument. Trace out the path on a domains map as shown in Figures 7 10 and 12-14. Now each converter corresponds to a line segment on the domains map. The conversion errors can be assessed by identifying and studying the reference source and difference detector for each converter. Modifications to instruments can be made by exchanging equivalent converters or by adding appropriate line segments to the instrument's domains map where new domains are to be included. New systems can be designed by completing a chart like Figure 16 for the devices available and comparing all the possible routes between the desired input and output domains.

Readers' comments, criticisms, and discussion on the concepts described in this article are welcomed. The author gratefully acknowledges the many helpful discussions he had with Dr. Howard Malmstadt, Dr. Stanley Crouch, Jim Ingle, and his graduate students during the evolution of these ideas.

\section{References} domain and intradomain conversions among these 11 domains and subdomains. However, direct converters for most of these transitions are rare or unknown. Thus the number of categories required to encompass the common converters is not unwieldy.

The domains map is a very convenient means of organizing domain con- verter categories. Figure 16 shows 21 put and output domains. Examples of devices for each listed domain transition are given in the accompanying table. This map and table clearly show what direct transitions are possible and which specific devices will do them. In addition, Figure 16 can be used to obtain and compare many possible combinations of devices to achieve a given transition by following connecting paths. Thus voltage-to-parallel digital converters could be mide by paths $3-$ $6-12,7-12,4-9-12,11-13$, and 18-15. These five trpes of $A / D$ converters are all currently marketed.

For any required conversion, that categories of converters arranged by in-
(1) H. V. Malmstadt and C. G. Enke "Digital Electronics for Scientists." W A. Benjamin, New York, N. Y., 1969.

(2) D. Hoeschele Jr., "Analog-to-Digitalto-Analog Conversion Techniques," Wiley, New York, N. Y., 1968.

(3) H. V. Malmstadt and C. G. Enke, "Computer Logic," W. A. Benjamin, New York, X. Y., 1970. 